\title{
Optimization of the quintic equation of the state based model for the calcu- lations of different thermodynamic properties
}

\author{
${ }^{1}$ Antoni Kozioł, Radosław Wiśniewski \\ Technical University of Wroclaw, Faculty of Chemistry, Division of Chemical and Biochemical Processes, Norwida 4/6, \\ 50-373 Wroclaw, Poland, e-mail: Antoni.Koziol@pwr.wroc.pl, Radoslaw.Wisniewski@pwr.wroc.pl \\ ${ }^{1}$ Corresponding author
}

\begin{abstract}
Different thermodynamic properties (the vapour density, the liquid density and the saturation pressure) were calculated by the model based on the Nakamura-Breedveld-Prausnitz equation of state (NBP EOS). Since the original form of the NBP EOS often generates inaccurate results for liquids, it was modified to describe this phase better. The calculations were realized in the subcritical region. So far, the temperature-dependent NBP EOS parameters have been obtained by special correlations. Their constants were fitted to a lot of experimental data. In this paper the equation of state temperature-dependent parameters were obtained by a new method which was based at piecewise cubic Hermite interpolating polynomials (PCHIPs). In the proposed method some experimental data (called the key ones) were used, thus reducing the experimental effort. Seven substances were chosen for the test calculations. Each of them is common in industry. The calculation results were compared with the experimental data. The new method has made an accurate description of vapour-liquid equilibrium for the considered pure substances over a wide temperature range possible.
\end{abstract}

Keywords: NBP equation of state, vapour-liquid equilibrium, cubic Hermite polynomials.

\section{INTRODUCTION}

Accurate thermodynamic data are necessary for the designing and optimization chemical engineering processes, such as distillation, heat exchange, adsorption or extraction. On the other hand, their experimental determination is labour-absorbing and time-consuming. So, it is very helpful to have a mathematical model, which can produce the required system properties.

The mathematical model should have theoretical fundaments and it can't be too complicated. It should describe both the normal systems and the highly non-ideal ones. The number of empirical constants must be as low as possible and they ought to be obtained by the use of a minimal number of experimental points.

At the moment, no thermodynamic models accurately predict all the properties of the highly non-ideal systems under all conditions. The available models are mainly based on cubic equations of the state or activity coefficients.

The models based on cubic equations of state, such as Peng-Robinson EOS ${ }^{1}$, Soave-Redlich-Kwong EOS $^{2}$ or Elliott-Suresh-Donohue EOS $^{3}$, may be used for the phase equilibrium and other properties calculations for normal systems, especially the hydrocarbons ones ${ }^{4}$. In order to describe the highly non-ideal systems many temperaturedependent empirical constants are required and their number increases with increasing the number of system components ${ }^{4}$. In addition, cubic equations of state usually describe the liquid phase poorly ${ }^{4}$.

The models based on activity coefficients ${ }^{5,6}$ can be used for the calculations of the phase equilibrium for the highly non-ideal systems but they are not sufficiently universal. They are thermodynamic approximations and other system properties, such as liquid density or speed of sound cannot be calculated. This class of models includes the empirical constants, which have to be fitted to many experimental data.
Thermodynamic properties can also be obtained by a molecular simulation technique ${ }^{7,8}$. The calculations reveal that these models are able to give accurate results but they are still too computationally intensive for the industrial use. This class of models is rapidly developing.

There are a lot of equations of state of degree higher than three. They have been known for a long time and their effective use has been possible since the latest computer technology development. They describe the liquid phase much better than the cubic EOS and the models based on them don't have the limitations that are common for the activity coefficients models.

The NBP EOS ${ }^{9}$ is the quintic one. This is a perturbedhard-sphere equation of state. It was given for gases and their mixtures containing highly polar molecules (such as water, ammonia or sulfur dioxide) and the non-polar or slightly polar molecules (such as hydrogen, argon, nitrogen, carbon monoxide, methane, ethane, ethylene, propane, propylene, hydrogen sulfide or carbon dioxide) $)^{9}$. In order to describe the supercritical fluids better, the NBP EOS was successfully modified by Koziod ${ }^{10}$. In this work, the NBP equation of state will be applied to describe the subcritical region.

Seven systems were chosen for the test calculations (water, methanol, isobutane, cyclohexane, trichlorofluoromethane, 1,1,1,3,3,3-hexafluoropropane and toluene). The experimental vapor-liquid equilibrium (VLE) data were taken from ref. ${ }^{11}$.

THE NAKAMURA-BREEDVELD-PRAUSNITZ EQUATION OF STATE

$$
\begin{aligned}
& \text { The NBP EOS is': } \\
& Z=\frac{1+\eta+\eta^{2}-\eta^{3}}{(1-\eta)^{3}}-\frac{a}{R T(v+c)} \\
& \eta=0.25 \frac{b}{v}
\end{aligned}
$$


where $Z$ is a compressibility factor, $\eta$ is the reduced density, $v$ is the molar volume, $T$ is the temperature, $R$ is a gas constant, $a, b$ and $c$ are the NBP EOS parameters. Parameters $a$ and $b$ are functions of temperature:

$a=\alpha+\frac{\beta}{T}$

$\log b=-\gamma+\delta T$

The values of constants $\alpha, \beta, \gamma, \delta$ and $c$ for water in the temperature range $653-1073 \mathrm{~K}$ are $0.31722 \mathrm{~Pa} \cdot \mathrm{m}^{6} \cdot \mathrm{mol}^{-2}, 117.71$ $\mathrm{Pa} \cdot \mathrm{m}^{6} \cdot \mathrm{mol}^{-2} \cdot \mathrm{K}, 1.5589,0.593 \mathrm{e}^{-4} \mathrm{~K}^{-1}$ and $1 \mathrm{e}-5 \mathrm{~m}^{3} / \mathrm{mol}$.

\section{THE MODIFICATION OF THE NAKAMURA- BREEDVELD-PRAUSNITZ EQUATION OF STATE FOR THE SUPERCRITICAL FLUIDS DESCRIPTION}

Kozioł $^{10}$ indicated that much better results for the supercritical fluids can be obtained by the replacement of equation (4) with the linear dependence and by the assumption that parameter $\mathrm{c}$ is also the function of temperature:

$a=a_{0}+\frac{a_{1}}{T}$

$b=b_{0}-b_{1} T$

$c=-c_{0}+\frac{c_{1}}{T}$

where $a_{0}, a_{1}, b_{0}, b_{1}, c_{0}$ and $c_{1}$ are fitted empirical constants. They can be determined by finding the minimum of the following objective function $F$ :

$F=\frac{1}{n} \sum_{i}^{n} \frac{\left|p_{i, E O S}-p_{i, \exp }\right|}{p_{i, \exp }} 100 \%$

where $p$ is a pressure, $n$ is a number of the experimental points. The values of constants $a_{0}, a_{1}, b_{0}, b_{1}, c_{0}$ and $c_{1}$ for water in the temperature range $380-2000 \mathrm{~K}$ are ${ }^{\mathbf{1 0}} 0.17336$ $\mathrm{Pa} \cdot \mathrm{m}^{6} \cdot \mathrm{mol}^{-2}, 213.130 \mathrm{~Pa} \cdot \mathrm{m}^{6} \cdot \mathrm{mol}^{-2} \cdot \mathrm{K}, 2.70 \mathrm{e}-5 \mathrm{~m}^{3} / \mathrm{mol}$, $1.883 \mathrm{e}-9 \mathrm{~m}^{3} / \mathrm{mol} / \mathrm{K}, 8.0 \mathrm{e}-6 \mathrm{~m}^{3} / \mathrm{mol}$ and $11.00 \mathrm{e}-3 \mathrm{~m}^{3} \cdot \mathrm{K} /$ mol.

\section{THE MODIFICATION OF THE NAKAMURA- BREEDVELD-PRAUSNITZ EQUATION OF STATE FOR THE SUBRCRITICAL REGION DESCRIPTION}

So far, the NBP EOS has successfully described the different gases and their mixtures. In this paper, the possibilities of the liquid phase description were verified. Table 1 presents the critical temperatures and the sources of the equilibrium data for the considered systems. It was assumed (exactly the same as in ref. ${ }^{\mathbf{1 0}}$ ) that all three parameters of the NBP EOS are temperature-dependent. In the subcritical region the equation of state must be true for the vapour phase and the liquid one. In addition, for the vapour-liquid equilibrium the Maxwell rule is applied. For each experimental point ( $T=$ constans) a system of three equations with three unknown EOS parameters was solved:

$$
\left\{\begin{array}{l}
\frac{R T}{v_{v}} \frac{1+0.25 b / v_{v}+\left(0.25 b / v_{v}\right)^{2}-\left(0.25 b / v_{v}\right)^{3}}{\left(1-0.25 b / v_{v}\right)^{3}}-\frac{a}{v_{v}\left(v_{v}+c\right)}-p=0 \\
\frac{R T}{v_{l}} \frac{1+0.25 b / v_{l}+\left(0.25 b / v_{l}\right)^{2}-\left(0.25 b / v_{l}\right)^{3}}{\left(1-0.25 b / v_{l}\right)^{3}}-\frac{a}{v_{l}\left(v_{l}+c\right)}-p=0 \\
{\left[v_{v}-v_{l}\right]^{-1} \int_{v_{l}}^{v_{v}}\left[\frac{R T}{v} \frac{1+0.25 b / v+(0.25 b / v)^{2}-(0.25 b / v)^{3}}{(1-0.25 b / v)^{3}}-\frac{a}{v(v+c)}\right] d v-p=0}
\end{array}\right.
$$

where subscripts $v$ and $l$ denote the vapour and liquid phase, respectively. For the given experimental values of $v_{v}, v_{l}$ and $p$ the parameters $a, b$ and $c$ were calculated. The equations system (9) is a highly non-linear one. It was solved by the Gauss-Newton method ${ }^{\mathbf{1 2}}$. The tolerance of the solution was $1 \mathrm{e}-12$.

Figure 1 shows the NBP EOS parameters as a function of temperature. All the parameters are strongly temperature-dependent with the exception of the $b$ parameter for water. Next, the saturated pressure, the vapour density and the liquid density were calculated by using the NBP EOS parameters from solving the equations system (9). The mean errors of the calculations were expressed as follows:

$\Delta=\frac{1}{3 n} \sum_{i}^{n}\left[\frac{\left|p_{i, E O S}-p_{i, \exp }\right|}{p_{i, \exp }}+\frac{\left|v_{v, i, E O S}-v_{v, i, \exp }\right|}{v_{v, i, e x p}}+\frac{\left|v_{l, i, E O S}-v_{l, i, \exp }\right|}{v_{l, i, \exp }}\right] 100 \%$

For the considered substances the values of $\Delta$ were less than $0.1 \%$. So, the vapour-liquid equilibrium can be perfectly described by the NBP EOS.

The experimental temperature dependences of the NBP EOS parameters are different for different substances see Figure 1. This fact makes their effective approximation with the simple functions such as Equations (5), (6) and (7) impossible. If we use more complicated functions, the number of experimental constants will be very high. In addition, the same functions can't be used for all the components, because sometimes the temperature dependences of the NBP EOS parameters can differ a lot - see Figure 1b. In our modification the temperature dependences of the parameters $a, b$ and $c$ were described by the method based on the piecewise cubic Hermite interpolating polynomials. Instead of the fitted empirical constants the VLE experimental data were used and the same method was applied for the description of all the parameters for all the substances (which is a big model simplification). The number of the used VLE experimental data was as low as possible and they were called the key ones.

Figure 2 shows the experimental and the calculated temperature dependences of the NBP EOS parameters for methanol and trichlorofluoromethane. For the PCHIP method only key data were used - see Table 2 . There is a big difference between the temperature dependence of methanol $b$ parameter and the temperature dependence of the trichlorofluoromethane $\mathrm{b}$ parameter. Owing to that the use of two different correlations is necessary for a correct description. However, the PCHIP method is able to give accurate dependences for both methanol and trichlorofluoromethane. In addition, Figure 2 shows that Equations (5), (6) and (7) are wrong for the subcritical region. 

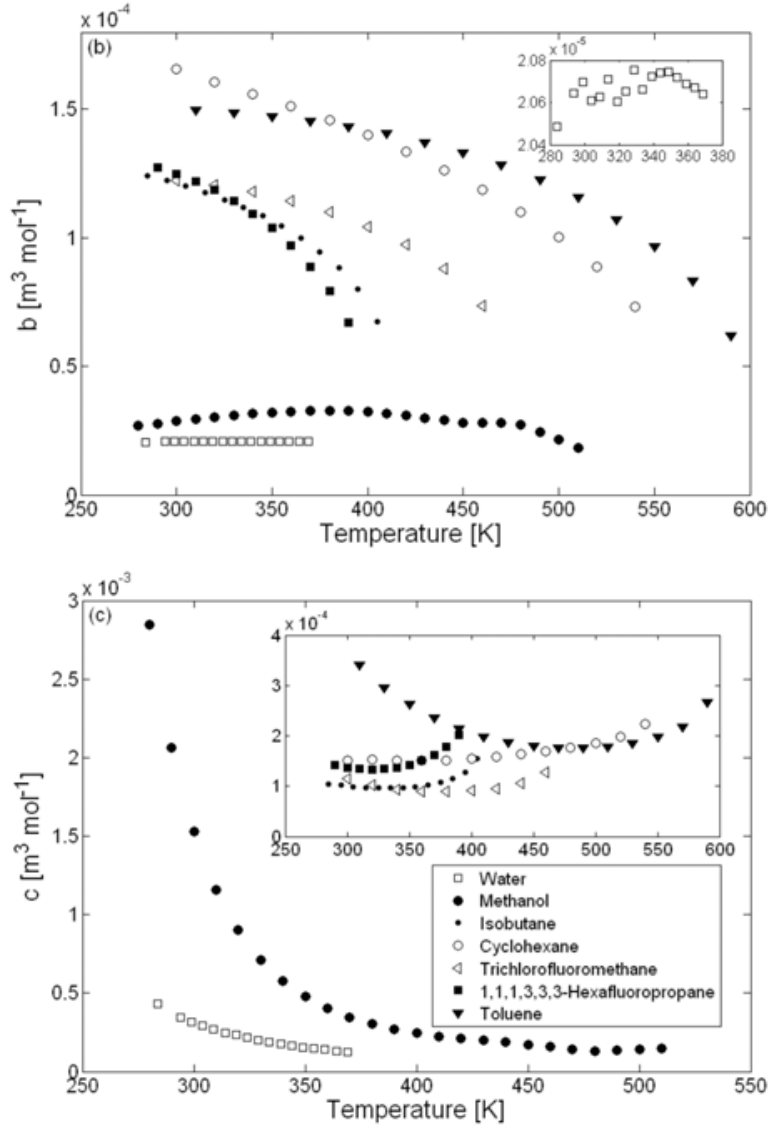

Figure 1. The experimental temperature dependences of the NBP EOS parameters
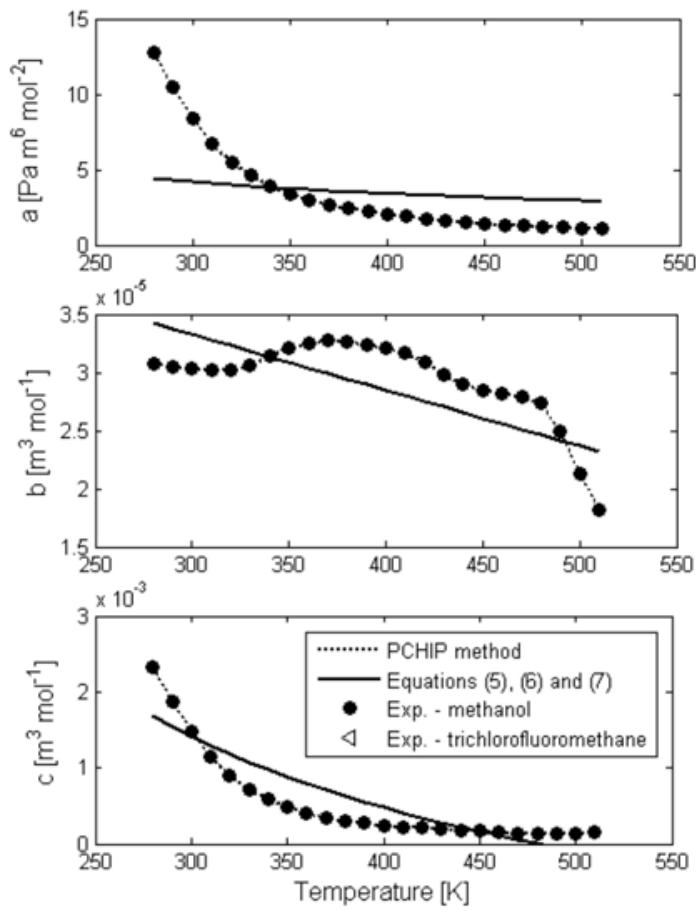

The Hermite form of polynomial consists of two points and two slopes of the interpolant. The considered subinterval is normalized to $(0,1)$ by using parameter $\lambda$. Then, the Hermite cubic polynomial $P$ is:

$P(\lambda)=H_{0,0}(\lambda) P_{0}+H_{1,0}(\lambda) D_{0}+H_{0,1}(\lambda) P_{1}+H_{1,1}(\lambda) D_{1}$

where $H$ is called the Hermite basis function, $P_{0}$ is a starting point at $\lambda=0, P_{1}$ is an ending point at $\lambda=1, D_{0}$ and $D_{1}$ denote the slopes of the interpolant at $\lambda=0$ and $\lambda=1$.

There are many possible ways to define the slopes of interpolant. In the used method the slopes were determined in such a way that the function values do not overshoot the data values, at least locally ${ }^{13}$. The applied method has been precisely described by Kahaner et al. ${ }^{14}$.

Table 2 shows the numbers of the key data that were used for the calculations. The $\Delta$ is the mean error (10) for the calculations results, which were obtained by using the PCHIP method. Next, the experimental temperature dependences were approximated by Equations (5), (6)

Table 2. The numbers of the key data and the mean errors of the VLE calculations

\begin{tabular}{|l|c|c|c|}
\hline Substance & $\begin{array}{c}\text { Number } \\
\text { of key data }\end{array}$ & $\Delta[\%]$ & $\Delta^{*}[\%]$ \\
\hline Water & 5 & 0.0649 & 8.33 \\
\hline Methanol & 9 & 0.758 & 12.4 \\
\hline Isobutane & 3 & 0.915 & 6.68 \\
\hline Cyclohexane & 3 & 1.24 & 14.5 \\
\hline Trichlorofluoromethane & 3 & 2.28 & 6.18 \\
\hline $\begin{array}{l}1,1,1,3,3,3- \\
\text { Hexafluoropropane }\end{array}$ & 3 & 0.997 & 5.65 \\
\hline Toluene & 4 & 2.03 & 70.3 \\
\hline
\end{tabular}
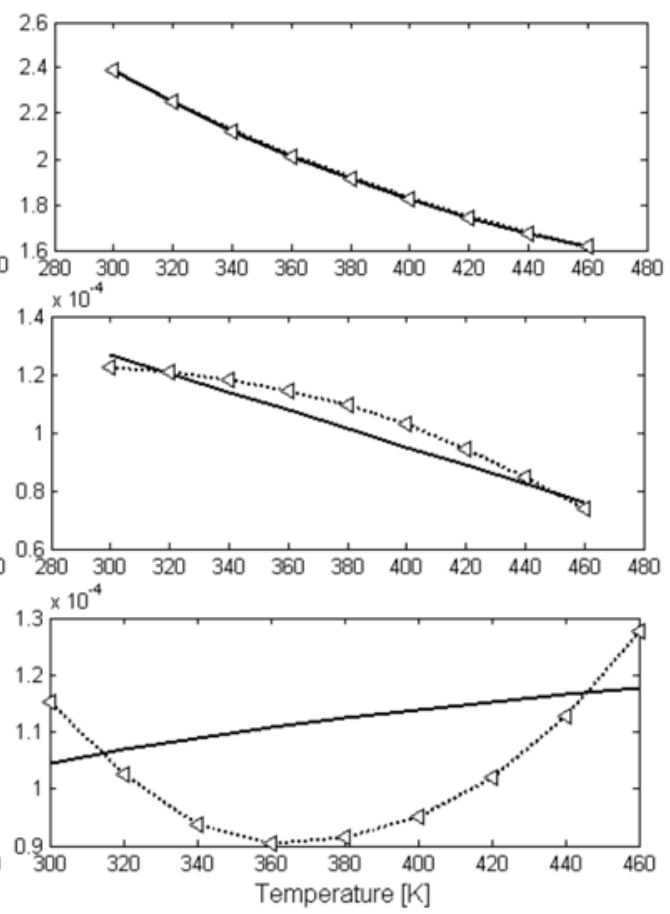

Figure 2. The experimental and calculated temperature dependences of the NBP EOS parameters for methanol and trichlorofluoromethane

Table 1. The critical temperatures and the sources of the equilibrium data

\begin{tabular}{|l|c|c|c|}
\hline Substance & Critical temperature $[\mathrm{K}]$ & Temperature range $[\mathrm{K}]$ & Source of VLE data \\
\hline Water & 647.096 & $284-369$ & 11 \\
\hline Methanol & 512.6 & $280-510$ & 11 \\
\hline Isobutane & 407.81 & $285-405$ & 11 \\
\hline Cyclohexane & 553.64 & $300-540$ & 11 \\
\hline Trichlorofluoromethane & 471.11 & $300-460$ & 11 \\
\hline $1,1,1,3,3,3-H e x a f l u o r o p r o p a n e$ & 398.07 & $290-390$ & 11 \\
\hline Toluene & 591.75 & $310-590$ & 11 \\
\hline
\end{tabular}



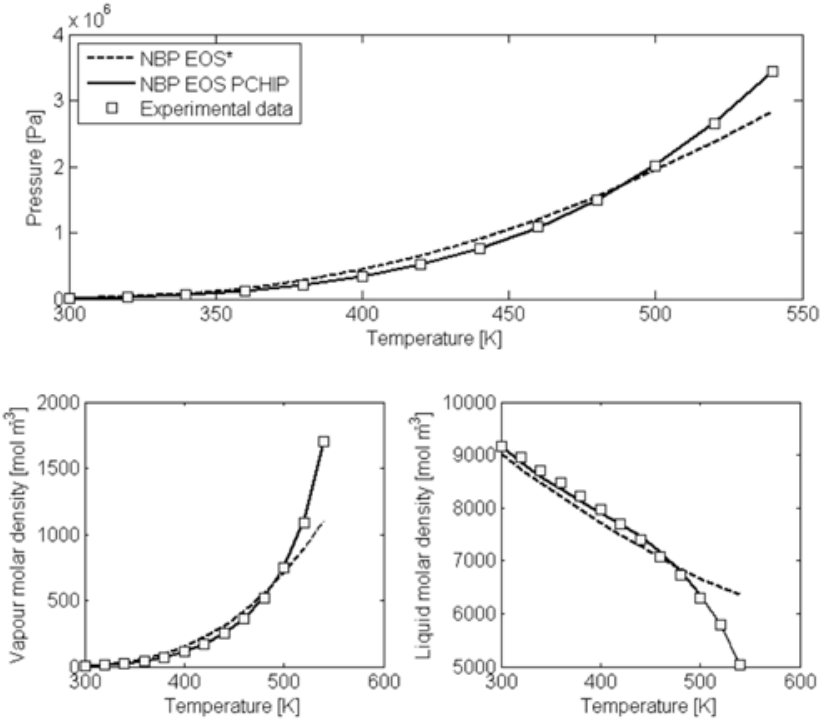

Figure 3. The VLE properties of cyclohexane

and (7). The ordinary least squares analysis was carried out. The $\Delta^{*}$ is the mean error (10) for the calculations which were obtained by using these functions. The errors $\Delta^{*}$ are much higher than for the PCHIP method. Notice that there are irregular temperature dependences of $b$ for water and methanol - see Figure 1b. The reason for this fact is not clear. This problem will be the object of future publication.

Figure 3 provides the calculated and experimental properties of cyclohexane. The symbol NBP EOS* denotes the calculations results obtained by using the best fitted Equations (5), (6) and (7). The NBP EOS PCHIP model gives accurate results for the considered range of temperature. The errors for the NBP EOS* model are much higher than the errors for the NBP EOS PCHIP one. The errors of the NBP EOS* model are the highest near the critical point.

\section{SUMMARY}

- Probably for the first time the NBP EOS temperaturedependent parameters have been obtained with the aid of the interpolation method. Instead of the classical correlations like Equations (5), (6) and (7) the piecewise cubic Hermite interpolating polynomials were used. The classical correlations must be fitted to a lot of experimental data and they aren't able to describe the atypical temperature dependences that exist for some systems, as it has been shown in Figure 2. The proposed method doesn't have the above disadvantages. It needs only some experimental key data and it can describe the atypical dependences, too.

- The calculations results showed that the NBP EOS and PCHIP methods correctly describe the vapor-liquid equilibrium for both the normal and the highly non-ideal pure substances.

- Generally speaking, the temperature dependences of the NBP EOS parameters for the supercritical region (the Equations (5), (6) and (7)) can't be used for the VLE calculations. For the considered systems the calculations errors $\Delta^{*}$ were high, as it has been shown in Table 2 .

- For calculations some thermodynamic properties, such as enthalpy of vaporization the derivatives of the NBP
EOS parameters at temperature are necessary. The proposed PCHIP method doesn't provide the temperaturedependent functions of the NBP EOS parameters but the required derivatives are expressed by the slopes of the interpolant. So, the proposed model can be also applied to other properties calculations and finally it ought to be extended to mixtures.

\section{SYMBOLS}

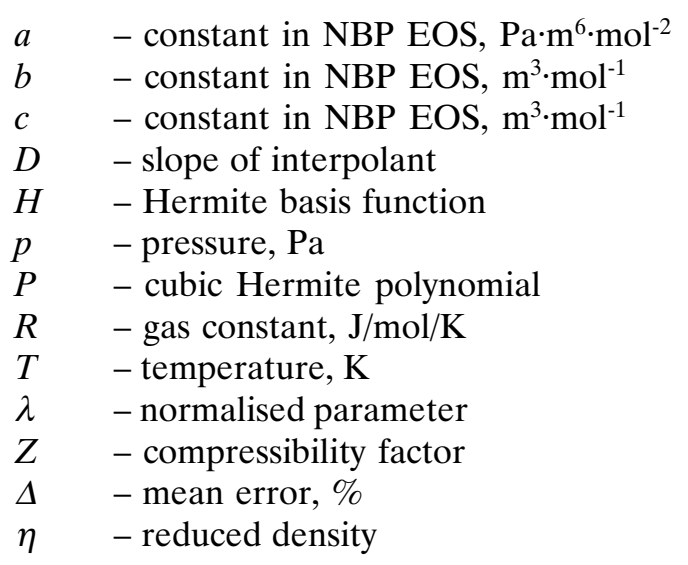

\section{SUBSCRIPTS AND SUPERSCRIPTS}

$v-$-denotes the vapour phase

$l \quad-$ denotes the liquid phase

$\exp -$ denotes the experimental value

$E O S$ - denotes the value calculated with the aid of EOS

\section{LITERATURE CITED}

1. Peng, Y. \& Robinson, B. (1976). A New Two-Constant Equation Of State, Ind. Eng. Chem. Fundam., 15, 58 - 64. 2. Soave, G. (1972). Equilibrium Constants From A Modified Redlich-Kwong Equation Of State, Chem. Eng. Sci., 27, 1197 - 1203.

3. Elliott, J., Suresh S. \& Donohue, M. (1990). A Simple Equation Of State For Nonspherical And Associating Molecules, Ind. Eng. Chem. Res., 29, 1476 - 1485.

4. Wisniewski, R. \& Koziol, A. (2005). Modelling Of Vapour-Liquid Equilibrium For Normal And Highly Non-Ideal Mixtures By Cubic Equations Of State And Mixing Rules, A. Chem. Proc. Eng., 26, 681 - 696.

5. Pazuki, G.R. \& Nikookar, M.(2006). A New Local Composition Model For Predicting Of Activity Coefficient And Solubility Of Amino Acids And Peptides In Water, Biochemical Engineering Journal, 28, 44 - 49.

6. I-Min, S., Wen-Lu, W. \& Ming-Chung, W. (2000). An Activity Coefficient Model For Predicting Salt Effects On Vapor-Liquid Equilibria Of Mixed Solvent Systems, Fluid Phase Equilibria, 170, 297 - 308.

7. Prasanna, K. Jog \& Walter, G. Chapman (1999). Application Of Wertheim's Thermodynamic Perturbation Theory To Dipolar Hard Sphere Chains, Molecular Physics, 97, 307 -319 .

8. Bureau, N., Jose, J., Mokbel, I. \& Dehemptinne, J.-C. (2001). Molecular Modeling Of Isomer Effects In Naphthenic And Aromatic Hydrocarbons. J. Chem. Thermo., 33, 1485 1498.

9. Nakamura, R., Breedveld, G. \& Prausnitz, J. (1976). Thermodynamic Properties Of Gas Mixtures Containing Common Polar And Nonpolar Components, Ind. Eng. Chem. Proc. Des. Dev., 15, 557 - 564. 
10. Kozioł, A. (2005). Application Of Various Equations Of State To Description Of Supercritical Fluids, Chemical And Process Engineering, 26, 727 - 734.

11. Lemmon, E., Mclinden, M. \& Friend, D. (June 2005). Thermophysical Properties Of Fluid Systems In Nist Chemistry Webbook, Nist Standard Reference Database Number 69, Eds. P.J. Linstrom And W.G. Mallard, , National Institute Of Standards And Technology, Gaithersburg Md, 20899 (Http://Webbook.Nist.Gov).

12. Coleman, F. \& Li, Y. (1994) On The Convergence Of Reflective Newton Methods For Large Scale Nonlinear Minimization Subject To Bounds, Mathematical Programming, 67, 189 - 224.

13. Moler, C., (2004) Numerical Computing With Matlab, Society For Industrial \& Applied Mathematics, 3, 8.

14. Kahaner, D., Moler, C. \& Nash, S. (1988) Numerical Methods And Software, Prentice Hall. 